Original Research Paper

\title{
Genetic Mutation Assessment of NPM1 Gene and Gene Expression of MDR1 in Iraqi Patients with Acute Myeloid Leukemia
}

\author{
${ }^{1}$ Ahmed Abduljabbar Suleiman, ${ }^{2}$ Ali Zaid Al-Saffar, \\ ${ }^{3}$ Tamadher Abbas Rafaa and ${ }^{4}$ Hasan Abdulwahab Jwad \\ ${ }^{1}$ Department of Biology, University of Anbar, College of Science, Iraq \\ ${ }^{2}$ Department of Molecular and Medical Biotechnology, \\ Al-Nahrain University, College of Biotechnology, Baghdad, Iraq \\ ${ }^{3}$ University of Anbar, University Headquarter, Iraq \\ ${ }^{4}$ Al-Nahrain University, College of Science, Baghdad, Iraq
}

Article history

Received: 07-12-2017

Revised: 20-12-2017

Accepted: 11-01-2018

Corresponding Author: Ahmed Abduljabbar Suleiman Department of Biology, University of Anbar, College of Science, Iraq

Tell: +964 7904774532

Email: ahmed.suleiman@uoanbar.edu.iq

\section{Introduction}

Acute Myeloid Leukemia (AML) is a type of heterogeneous disorder with a diversity of genetic abnormalities and cytogenetic alterations that provide a significant disease prognosis and determining therapy responsiveness (Liu et al., 2007). AML resulted from a series of frequent hematopoietic stem cell genetic aberration accumulated with age (Ding et al., 2012). Childhood AML generally occurs de novo (Hamed et al., 2011).

Nucleophosmin 1 (NPM1) mutations are most frequently identified (25-30\%) in AML patients, with female predominance. These mutations lead to the abnormal expression of NPM1, releasing the protein in the cytoplasm rather than the nucleus and stimulating

\begin{abstract}
Since its discovery, mutations in NPM1 have been frequently associated with a large number of Acute Myeloid Leukemia (AML) patients. The vast majority of genetic changes were previously detected in exon 12, however little information report mutations in Nuclear Export Signal region NES) of NPM1. Sequencing analysis included exon 2 and 3 for 75 Iraqi AML patients showed three SNPs, G/A792, G/A794 and G/A797 were SNPs were only detected in AML subjects in which single nucleotide variant was identified in exon 3 of $70 \%$ AML subjects (A/G1275 rs753788683) in addition, two SNPs (G/A635 and G/A660) within intron region of $80 \%$ of amplified NPMI gene. The correlation between MDRl gene over expression and resistance to chemotherapy treatment showed no significant differences in gene expression between newly diagnosed and first-course induction sertheless, significant decreases in CT value were recorded for both the second induction AML and AML consolidation patients with $p$ value indicating the induction of higher expression of $M D R 1$ gene by increasing the challenge of AML patients with chemotherapy regimen.
\end{abstract}

Keywords: AML, NPM1, Multidrug Resistance, Sequencing myeloid proliferation and leukemia development (Falini et al., 2007). NPM1 gene consists of 12 exons and located at chromosome 5q35. The NP Mmolecule contains distinct domains responsible for its multiple biochemical functions (Hingorani et al., 2000). The traffic of NPM between the nucleus and cytoplasm is highly regulated and mostly the regulation comes through the Nuclear Localization Signal (NLS) and Nuclear Export Signal (NES) motifs. NLS directs NPM from the cytoplasm to the nucleoplasm, which then translocates to the nucleolus through its nucleolar binding domain, mainly tryptophan residues 288 and 290 (Yu et al., 2006). NPM remains in nucleoli, though it contains highly conserved hydrophobic leucine-rich NES was encoded by exon 2 and 3 motifs within 
residues 94-102 and 42-49, which drive it out of the nucleus (Wang et al., 2005; Yu et al., 2006).

Most studied NPMI mutations were restricted to exon 12. All variants cause alterations at the Cterminus of the NPM leukemic mutants, within exon 12 region and this alteration is responsible for NPM dislocation into the cytoplasm, due to changes of tryptophan (s) 288 and 290 and creation of an additional NES. Thisprotein tends to be stable and accumulated in the cytoplasm (Suzuki et al., 2005). Limited studies were conducted in detecting mutations in NPM1 gene within exon 2 and/or exon 3 of AML patients. Both exons encoded for the $\mathrm{N}$-terminal portion of NPM1 protein which plays a major role in protein oligomerization and chaperone activities (Herrera et al., 1996) also this region encode the Nuclear Export Signals (NES) that prove protein accumulation in the cytoplasm (Kazem et al., 2011).

Within Iraqi population, leukemia considered one of the most common types of cancer after breast cancer and lung cancer. The annual incidence of leukemia in Iraq is about 728 per 10,000 and it affects males $57 \%$ more than females $43 \%$. As far as the prevalence is concerned, leukemia is the third most prevalent form of cancer after breast cancer in Iraqi population with annual significant increases each year (ICB, 2014).

On the other hand, treatment of leukemia considered as a challenge due to the development of resistance to chemotherapeutic agents. Assessing the drug resistance of leukemic cells is, therefore, an important aspect of treatment. One of the main mechanisms of resistance is rapid drug efflux mediated by various members of the ATP-binding cassette transporter super family, such as multidrug resistance gene $1(M D R 1)$, which encodes Pglycoprotein (Schaich et al., 2005). Therefore, the current study was aimed to detect possible mutation(s) within exon 2 and 3 in NPM1 gene and monitoring the expression of MDRl gene by comparing the expression before and after the exposure to chemotherapy.

\section{Materials and Methods}

\section{Patients and Samples}

The study consisted of 75 Iraqi AML patients (45 male and 30 female), all patients were above 18 years, with median age of 33 . Peripheral Blood samples (3-5 $\mathrm{mL}$ ) were collected from the patients during the period starting from December 2015 to March 2016, which were admitted to Baghdad Teaching Hospital (BaghdadIraq). All patients were diagnosed with AML based on updated French-American-British (FAB) classification (Vardiman et al., 2009).

Most patients were treated with chemotherapy. The induction chemotherapy regimens were, combined cytarabine plus adriamycin or combined vincristine plus doxorubicin or daunorubicin and All-Trans Retinoic Acid (ATRA) plus induction chemotherapy, depended on the subtype of AML. Accordingly, patients were categorized into four groups, newly diagnosed (No treatment started) 15 patients; 20 AML patients after first-course induction of chemotherapy; 20 AML patients after second induction and $20 \mathrm{AML}$ patients in consolidation. In addition, another 25 blood samples were collected from healthy volunteers and considered as controls. The current study was approved by the Board of Baghdad Teaching Hospital and the College of Biotechnology at Al-Nahrain University.

\section{Screening for NPM1 Gene Mutations}

DNA was extracted from both patients and health volunteers using SYNCTM DNA Extraction Kit (Genead, South Korea). Primers used for NPM1 gene amplification were: $\quad N P M X 1 \mathrm{~F}$ 5'TGTGAACTAAAGGCCGACAA-3', NPMX1R 5'CCACAGGGCTAGGTTCTGAG-3', NPMX2F5'AGCCCTTGTAAAAGGCATCG-3', NPMX2R 5'CCCAAGGGAAACCCTAGAAGG-3'.

Gene mutations flanking the region start from 2380 to $4140 \mathrm{pb}$ of NPM1 gene (Fig. 1), which represent exons 2 and 3 , were analyzed by amplifying the indicated region of the gene and analyzed on agarose gel $(2 \%)$ in presence of $100 \mathrm{bp}$ DNA ladder marker, followed by direct sequencing technique (Macrogen, South Korea). Sequencing products were compared with the information in gene bank of the National Center for Biotechnology Information (NCBI) for standard NPMI gene, using Mega software, version 6.

\section{MDR1 Expression in AML Patients}

The expression of MDRl transcripts was estimated by Real Time-qPCR. Primers were designed and supplied by BioNeer (South Korea) in a lyophilized form in the following sequence: MDR1F5'GGAGGCCAACATACATGCCT-3', MDRlR5'-CAG GGCTTCTTGGACAACCT-3'. Lyophilized primers were dissolved in a free DNase/RNase water to give a final concentration of $100 \mathrm{pmol} \mu \mathrm{L}^{-1}$; it was stored at $20^{\circ} \mathrm{C}$ until use. In addition a working solution of 50 pmol $\mu \mathrm{L}^{-1}$ of oligo (dT) primer was prepared using deionized distilled water. The real-time PCR experiment was designed in singleplex assay, the chemistry used in real-time qPCR was DNA-binding dyes SYBR Green1.

RNA extraction was carried out by using Total RNA Mini Kit (Blood/Culture Cell) Protocol System (Geneaid, South Korea). The purity of RNA was measured at 260/280 nm absorbance using Nanodrop. While Reverse transcription of total RNA to cDNA was achieved using RT-PCR PreMix kit (Bioneer, South Korea). Real-Time qPCR was carried out according to the designed amplification program. 


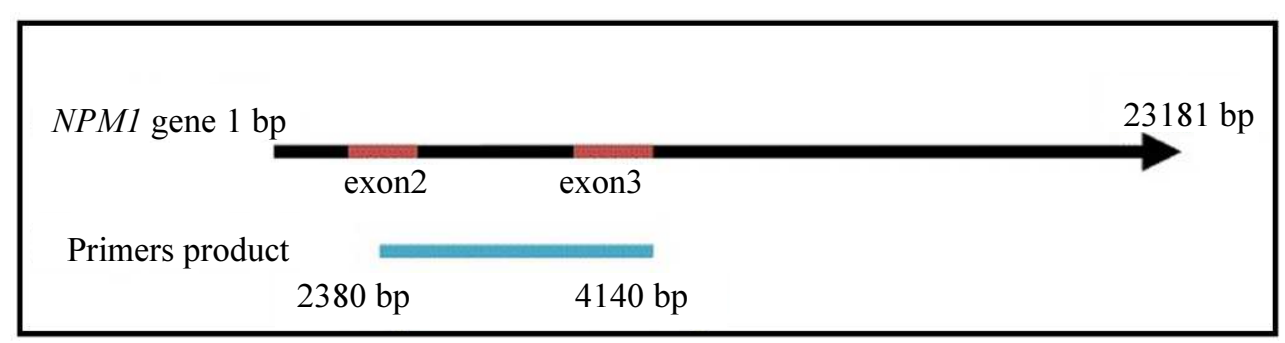

Fig. 1: The product of $N P M 1$ gene primers

\section{Data and Statistical Analysis}

Relative expression of MDR1 gene in AML patient and healthy control was calculated using the equation: Ratio $=2-(\Delta \Delta$ CT) (Livak, 2001). Statistical analysis was carried out using Graph Pad Prism (version 6). One way analysis of variance ANOVA (Duncan) and Chi-square were performed to test whether group variance were significant or not. Data were expressed as mean \pm standard error. $P$-values were considered to be statistically significant if $p \leq 0.01$.

\section{Results}

\section{NPM1 Gene Amplification}

The region of NPMI was amplified from the extracted DNA of both AML patients and healthy controls. Results illustrated in Fig. 2 shows the amplified products appeared as clear bands after electrophoresis on agarose gel $(2 \%)$. The product of NPMX1 primer (Fig. 2A) was a single clear band with molecular size of $1150 \mathrm{bp}$, this product represents about 2380 to $3447 \mathrm{bp}$ of amplified region and cover exon 2 from NPM1 gene, while the product of NPMX2 primer (Fig. 2B) was also a single band with molecular size of $1337 \mathrm{bp}$, which represent 2707 to $4140 \mathrm{bp}$ of amplified region that covered exon 3.

\section{NPM1 Gene Sequencing and Mutations}

The complete nucleotide sequence of PCR products (2380 to $4140 \mathrm{bp}$ ) was analyzed to determine any genetic changes in this region. Results of sequencing that illustrated in Table 1 shows the genetic variations and frequencies of mutations associated with the studied AML cases.

Three different Single Nucleotide Polymorphism (SNP) G/A792, G/A794 and G/A797 were detected in the intron region. G/A792 was found in 68 of 75 AML patients $(90.6 \%), \mathrm{G} / \mathrm{A} 794$ was found in 60 of 75 AML patients $(80 \%)$, while G/A797 was found in all AML patients. All of the three were detected in 17 of 25 healthy controls $(68 \%)$. These SNPs were not before correlated with mutant NPMI Iraqi AML patients and are first described. All statistical analysis that compares between AML cases and healthy controls were summarized in Table 2.

Single nucleotide variant was identified in exon 3 that encode NES, it was found in 53 of 75 AML patients $(70.6 \%)$. This variant was not detected in healthy subjects. Based on statistical analysis, this NPM1 mutation was significantly associated with AML $(p=0.0022)$. In addition, single nucleotide variant was observed in two sites G/A635 and G/A660 within intron region that were identified in $80 \%$ of AML patients. These SNPs were significantly correlated with mutant NPM1 $(p=0.0153)$. No genetic variation was observed in exon 2 during sequences analysis of amplified NPM1 gene.

Amino acids sequence encoded by exon 3 of NPM1 gene in AML patients were examined and compared with the reference sequence recorded in NCBI. Results (Table 3) shows the alignment between patients and reference sequence ID: NP-002511.1, Length: 294, Range 1: 47 to 87 nucleophosmin isoform 1 Homo sapiens.

$\begin{array}{lll}\text { Query } & 1189 & \text { VSLGAGAKDELHIVEAEAMN } \\ & & \text { YEGSPIKVTLATLKMSVQPTV } \\ & & \text { VSLGAGAKDELHIVEAEAMN } \\ & & \text { YEGSPIKVTLATLKMSVQPTV } \\ \text { Sbjct } & 47 & \text { VSLGAGAKDELHIVEAEAMN } \\ & & \text { YEGSPIKVTLATLKMSVQPTV }\end{array}$

1311

The position of amino acid (threonine, T) related to the expected rs753788683 SNP occurred in exon 3 at position 75 within the reference sequence. The results showed no changes in the sequence of amino acids (100\% similarity) between AML sequence and the reference sequence. The codon that encodes the threonine at position 75 was ACA. The SNP was a base substitution mutation that changed $A$ to $G 1275$ (rs753788683) causes transversion in the codon from ACA to ACG, however, this altered codon encoded the same amino acid threonine, so the resulted mutation was silent and not affected the amino acid sequence. 


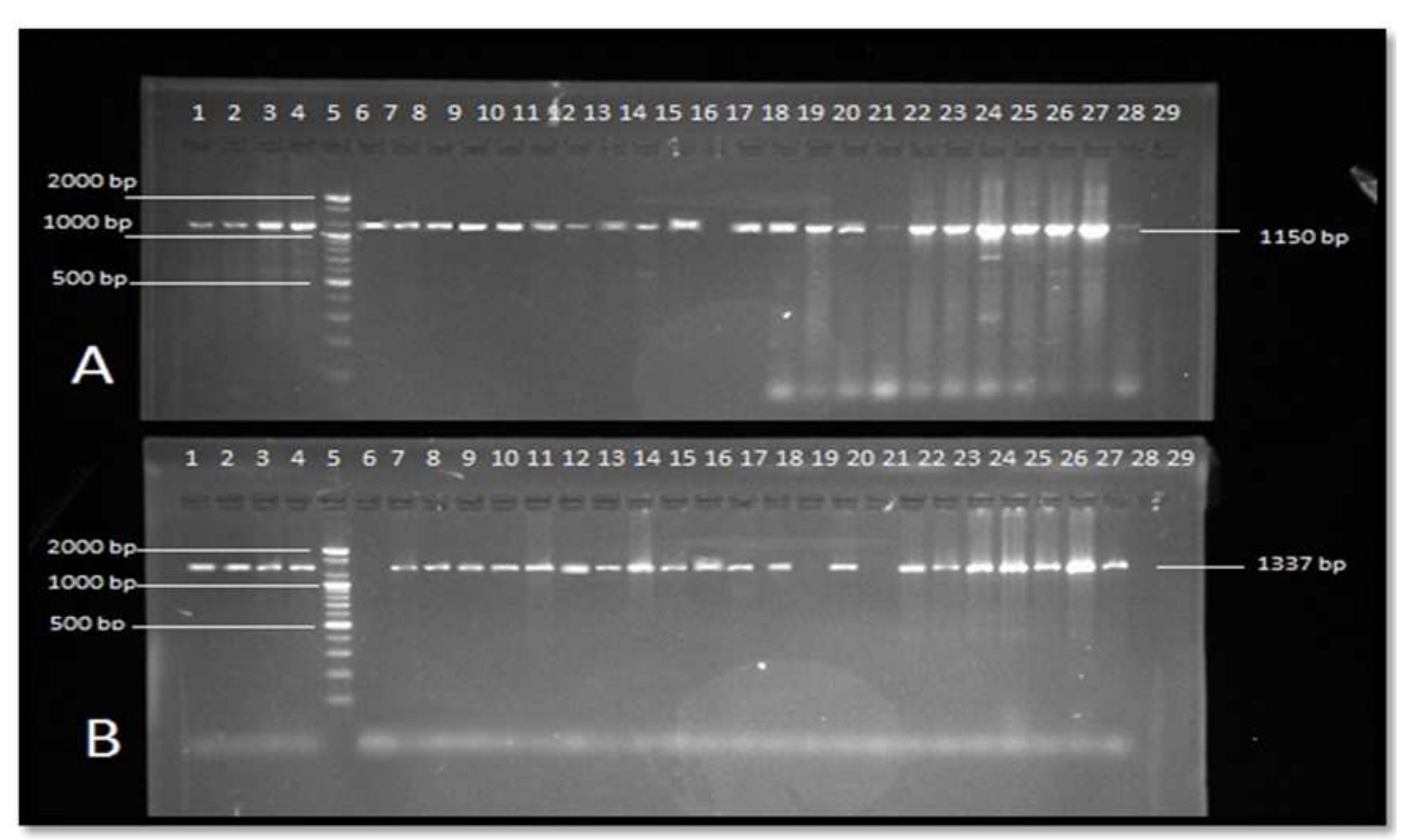

Fig. 2: PCR products of $N P M 1$ on agarose gel $(2 \%)$ ran for $1 \mathrm{~h}$ at $70 \mathrm{~V}$ in the presence of 100 bp DNA Ladder marker. (A): PCR products of NPMX1 primer (B): PCR products of NPMX2 primer. Lane (1-4) Healthy Control. Lane (5) DNA Ladder Marker. Lane (6-29) Patient Bands

Table 1: Genetic variation in NPMI gene

\begin{tabular}{llllll}
\hline Type & Substitution & Variation & Position & Healthy (25) & AML (75) \\
\hline SNP & G/A & Intron & 792 & 17 & 68 \\
SNP & G/A & Intron & 794 & 17 & 60 \\
SNP & G/A & Intron & 797 & 17 & 75 \\
SNP & G/G & Exon 3 & 1275 & 0 & 53 \\
SNP & G/A & Intron & 660 & 0 & 60 \\
SNP & G/A & Intron & 635 & 0 & 60 \\
\hline
\end{tabular}

Table 2: Statistical analysis of NPM1 mutations

\begin{tabular}{|c|c|c|c|c|}
\hline Variant & $P$ value & Sig. & OR & $\mathrm{CI}$ \\
\hline G/A792 & 0.006 & $* *$ & 4.571 & $1.014-20.6$ \\
\hline $\mathrm{G} / \mathrm{A} 794$ & 0.216 & NS & 1.882 & $0.683-5.185$ \\
\hline $\mathrm{G} / \mathrm{A} 797$ & $<0.0001$ & $* * * *$ & 73.34 & $4.036-1333$ \\
\hline $\mathrm{A} / \mathrm{G} 1275$ & 0.0022 & $* *$ & 0.0466 & $0.001-1.955$ \\
\hline $\mathrm{G} / \mathrm{A} 660$ & 0.0153 & $*$ & 0.076 & $0.0017-3.26$ \\
\hline $\mathrm{G} / \mathrm{A} 635$ & 0.0153 & $*$ & 0.076 & $0.0017-3.26$ \\
\hline Sig: Significance. & OR: Odd Ratio. & \multicolumn{3}{|c|}{ CI: Confidence of Interval (95\%) } \\
\hline
\end{tabular}

Table 3: Alignment of NPMlexon 3 amino acids sequence between AML sample and reference sequence

\begin{tabular}{|c|c|c|c|c|c|c|}
\hline Score & Expect & Method & Identities & Positives & Gaps & Frame \\
\hline 84.3 bits(207) & $3 \mathrm{e}-17$ & Compositional matrix adjust. & $41 / 41(100 \%)$ & $41 / 41(100 \%)$ & $0 / 41(0 \%)$ & +1 \\
\hline
\end{tabular}

\section{MDR1 Gene Expression Assessment in AML Patients}

The expression of MDRl gene in AML patients was achieved by RT-qPCR. The patients were categorized depending on their chemotherapy induction, as newly diagnosed (No treatment started), first-course induction of chemotherapy, second induction and AML patients in consolidation. The healthy control (untreated) used as calibrator.

The result of amplification reaction was summarized in the Fig. 3. The amplification reaction increase dependent on mRNA copy of MDRl gene in each group that treated with chemotherapy. Also, real-time PCR 
products showed only a single melting temperature peak was observed for each reaction Fig. 4, thus suggesting nonspecific amplification occurred, like primer dimmer, DNA contamination and other non-specific bindings.

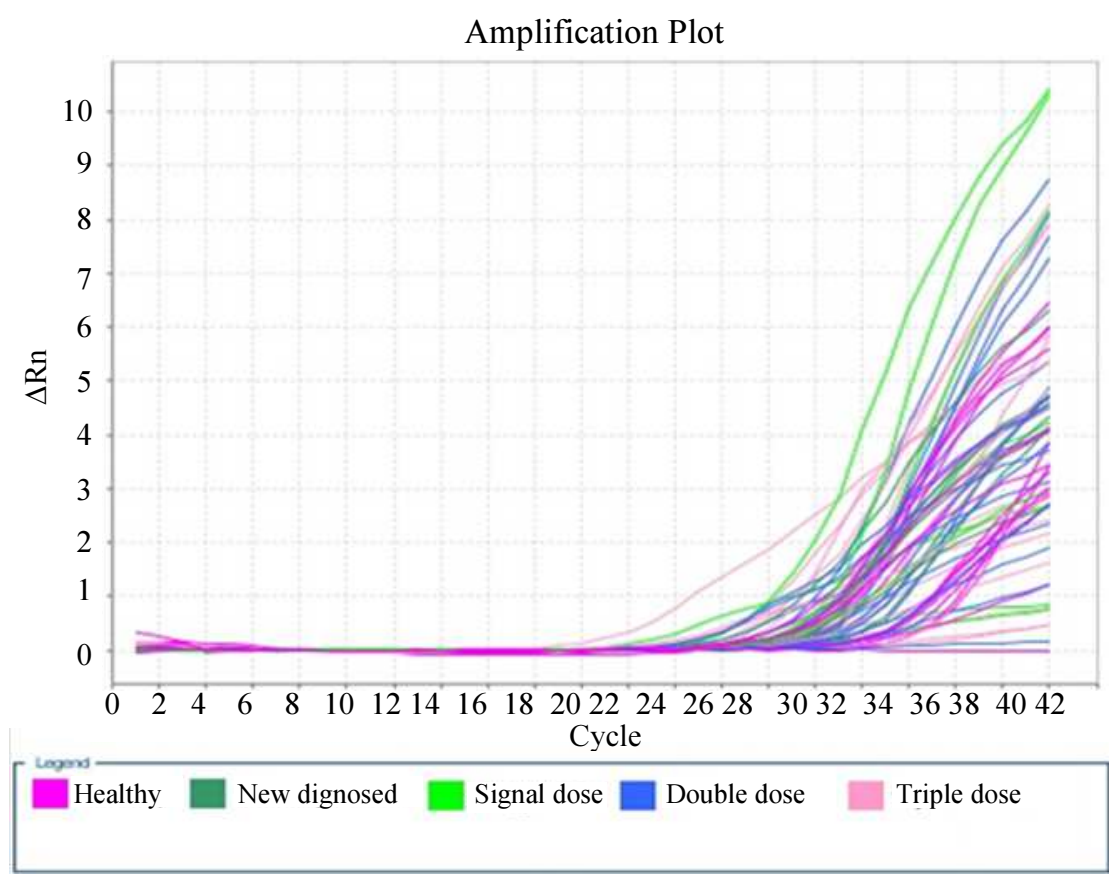

Fig. 3: Real-Time qPCR amplification plot of $M D R I$ gene

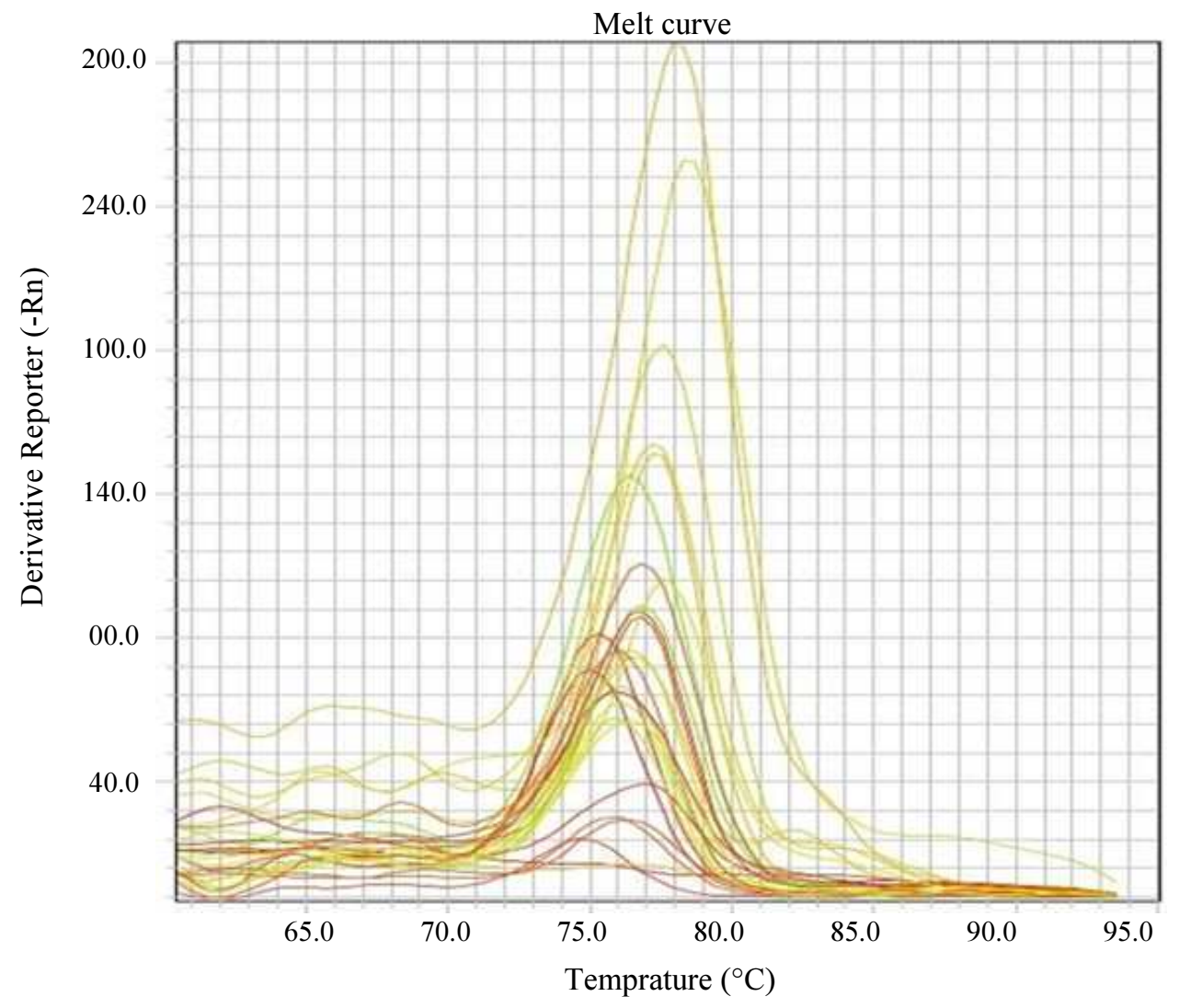

Fig. 4: Real-Time qPCRmelting curve of tested samples 


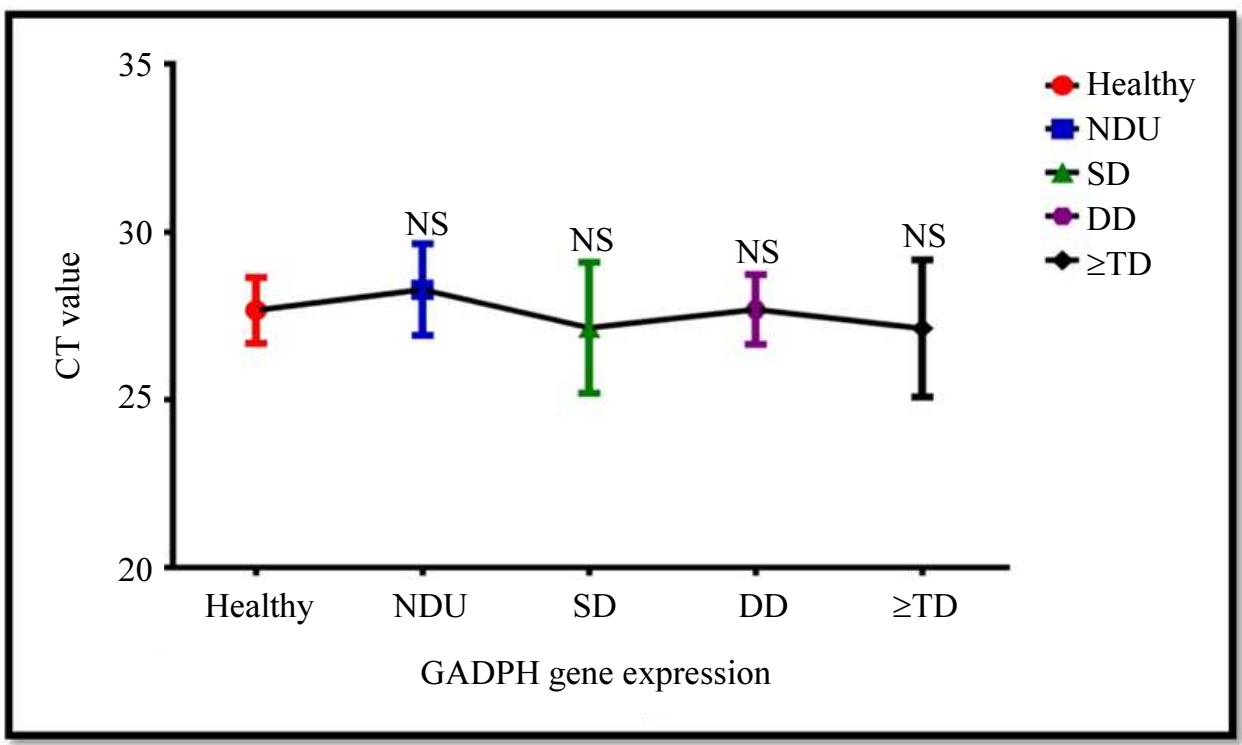

Fig. 5: GADPH endogenous control gene expression in healthy controls and AML patients. New Diagnoses Untreated (NDU), Single Dose (SD), Double Dose (DD), Triple Dose (TD), Non-Significant (NS)

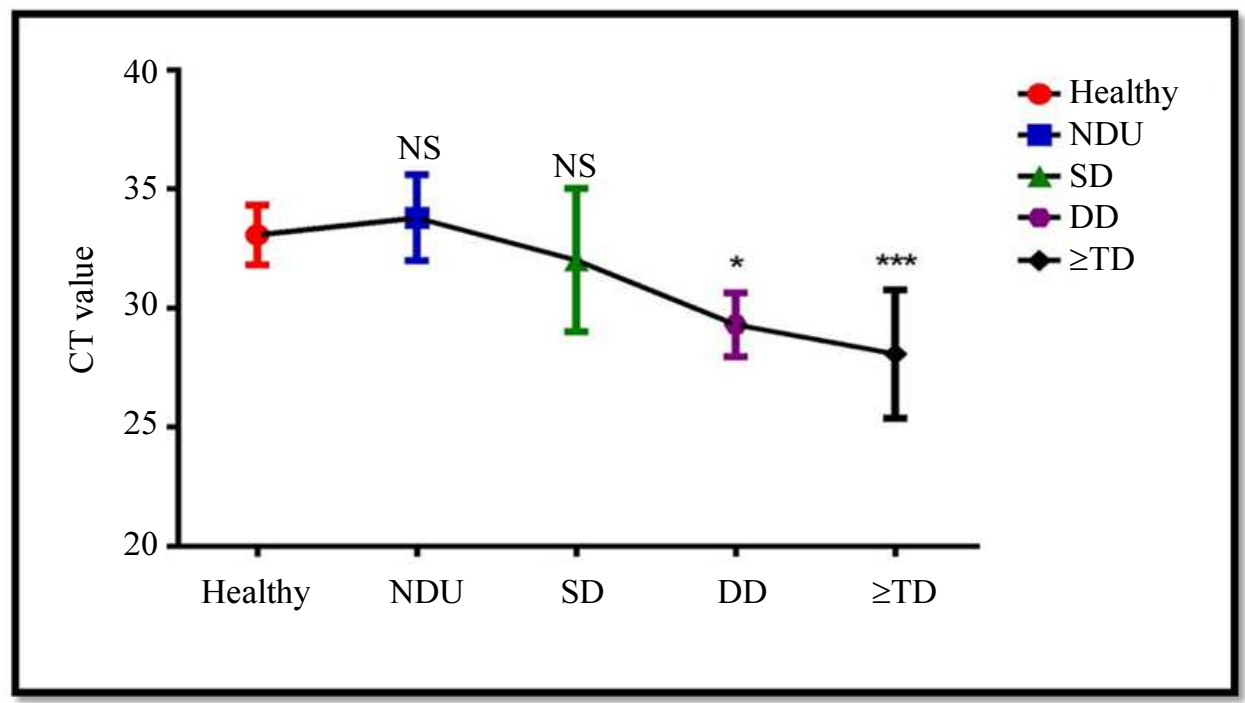

Fig. 6: The expression of MDRl gene in AML patients and healthy controls. New Diagnoses Untreated (NDU), Single Dose (SD), Double Dose (DD), Triple Dose (TD), Non-Significant (NS)

The expression MDRl gene was compared between the different groups (Fig. 5 and 6). The Y-axis represents the mean CT value of expression while $\mathrm{X}$-axis represents the five group of analysis. Result (Fig. 5) showed that the expression of the standard GADPH gene had no significant differences between healthy control and the four groups of AML patients. No significant differences in MDRl expression between the newly diagnosed AML group and first course AML patients as compared with controls (Fig. 6). Nevertheless significant decreases in CT value were recorded for both the second induction AML group and AML consolidation group with $p$ value of 0.0258 and 0.0007 , respectively, as compared with healthy controls, indicating the induction of higher expression of $M D R 1$ gene by increasing the challenge of AML patients with chemotherapy regimen.

Further assessment of different MDRl gene expression in AML patients was done by calculating the relative ratio for testing patient groups as compared with healthy controls (Fig. 7). Results showed no significant coloration between healthy and newly diagnosed since the relative expression ratio was -0.34 fold $(p=0.1591)$, therefore both groups have similar MDR1 gene expression. 


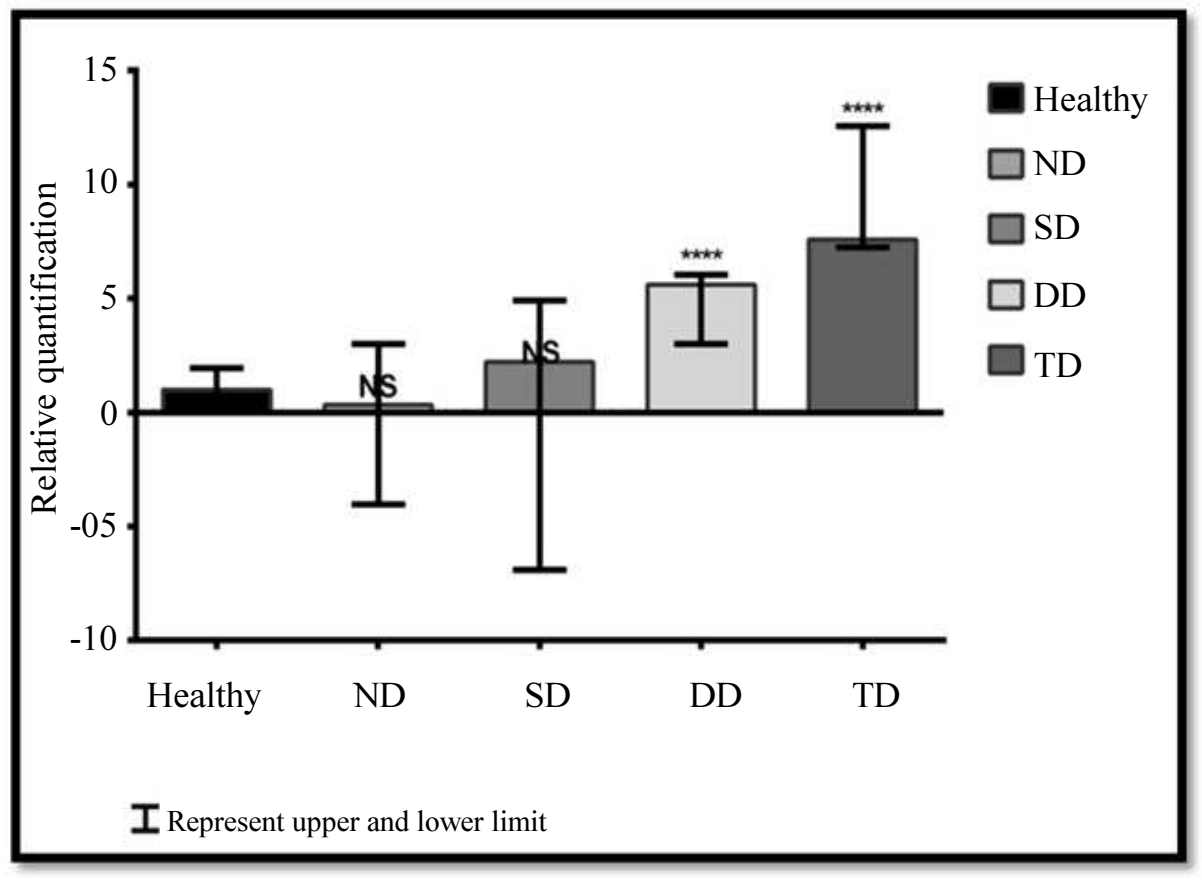

Fig. 7: Relative quantification of different AML groups compared with healthy controls (calibrator). New Diagnosed (ND), Single Dose (SD), Double Dose (DD), Triple Dose (TD), Non-Significant (NS)

This suggested no induction in gene expression. Also no significant differences between healthy calibrator and single chemotherapy AML induction group with relative expression ratio of 2.22 fold, $p$ value 0.2222 . Upregulation of MDR1 gene was clearly observed in AML patients with second induction by 5.6 fold increase with significant differences as compared with calibrator $(p<0.0001)$, in addition MDRl high gene expression, was also observed in AML consolidation group with a relative ratio of 7.58 fold increase and significant differences comparing with control group $(p<0.0001)$.

\section{Discussion}

The exon 3 and exon 2 encode the N-terminal region of NPM1 protein. In this study a mutation in exon 3 (rs753788683) was recorded, even though this mutation was not affected on amino acid sequences. Mutations in intron region not affecting on amino acids sequences but may affect gene expression. The N-terminal portion of NPM1 protein was important for oligomerization and chaperon activities (Herrera et al., 1996) and any mutation in this region cause defect in portion function. NPM1 mutations are restricted to exon 12 of AML patients (Kazem et al., 2011) and all mutations tend to defect NLS signal and modify it into new NES signal in exon 12, the NES signal was important in mutant NPM1 protein to accumulated the protein in the cytoplasm (Chou et al., 2006). More than 40 molecular variants of NPM1 mutations have been described in AML patients
(Tan et al., 2008). The most common mutation (socalled mutation A12) duplicates a TCTG tetra nucleotide (Szankasi et al., 2008). Mutations B and D are observed in about 10 and $5 \%$ of cases, respectively; other mutations are very rare (Falini et al., 2007). In addition the N-terminal region contains high conserved hydrophobic leucine-rich NES motifs (Wang et al., 2005; Falini et al., 2009), therefore it is very difficult that mutations to occur in this region. The region that encodes for NES was important for protein function and any genetic variation in NES signal may attribute in increasing or decreasing protein function.

The goal of chemotherapy treatment is to achieve a complete remission of AML and then to prevent relapse in post-remission therapy. In the last few years, there have developing changes in the diagnosis and treatment of AML based on molecular genetic assessment of $M D R 1$ expression for designing novel curative regimens that reversing regulation of drug-resistant phenotype of AML cells (Cianfriglia, 2013). Response to treatments is affected by many factors according to risk categories associated with morphological features, genetic criteria and age (Almeida and Ramos, 2016; Webber et al., 2008).

Longley and Johnston (2005), stated that such findings correlate with the concept of AML being an intrinsically resistant disease and that such up-regulation could be acquired during induction treatment. This support our study that higher expression of MDR1 significantly observed in group two 5.6 fold and group three 7.58 fold and in another hand the non-difference in 
$M D R 1$ gene expression between the calibrator and newly diagnosed ratio -0.34 fold. Up-regulation of MDR 1 after induction has been reported by others. showed in vitro study, the MDRl and MRP1 (drug resistance proteins) mediated in multidrug resistance of human leukemic cells through the mechanism of resistance to doxorubicin-induce cell death in human HL60 AML cells, they indicated the continuous exposure of leukemic cells to stepwise increasing concentration of doxorubicin resulted in the selection of HL60/DOX cells, which expressed about 10.7 fold resistance as compared to parental sensitive (Baran et al., 2007).

The existence of Cancer Stem Cells (CSCs) has been proposed as a potential mechanism ofdrug resistance and a potential therapeutic target (Gupta et al., 2009). CSCs are a putative population of malignant cells within a tumor that possess the characteristics associated with normal stem cells. In this theory's most elaborate conception, CSCs can self-renew and divide by asymmetric division, reconstituting the heterogeneity of the original tumor. CSCs are also considered to be highly resistant to chemotherapeutic agents (Honoki, 2010). In this model, chemotherapy kills most of the cancer cells that are some what more differentiated and do not have long-term self-renewal capacity. When therapy is discontinued, the CSCs repopulate cancer, there by mediating disease relapse. In contrast, molecular remission after consolidation has important prognostic value. This implies that despite $C R$, in these patients a number of cancer cells survive treatment and can grow out to cause a relapse. The increased MDR1 expression after induction may cause early relapse and progression disease. These studies agreed with our finding that revealed in one case showed complete remission after induction then relapsed occurred after consolidation.

In addition to the involvement of the ATP-Binding Cassette $(\mathrm{ABC})$ family in the efflux and reduction of intracellular drug concentration in malignant conditions, it has recently been shown that these proteins have a role in transportation of prostaglandins, leukotrienes, Sphingosine-1-Phosphate (S1P) and Platelet-Activating Factor (PAF) out of the cells. These lipids cause activation of different signaling pathways, which results in cell proliferation, migration, survival and activation of angiogenesis, metastases and the escape of leukemic cells from immune surveillance (Fletcher et al., 2010). Various ABC transporters are specific to lipids. MDR1 is involved in the efflux of PAF; a PAF molecule activates its G-protein-coupled receptor, PAER and induces up regulation of the anti-apoptotic proteins $\mathrm{Bcl}-2$ and Bcl-XL. These molecules prevent programmed cell death and cause expansion of resistant cells (Raggers et al., 2001). Therefore MDR1 protein has another function that important in AML proliferation. When the AML patients take chemotherapy the expression of MDR1 gene will increase, so the function of MDRl gene will increase cell proliferation of leukemia cells. This suggested that when patient taken chemotherapy then relapsed occurred after consolidation, the chemotherapy will be ineffective in our patient and also increase the severity of AML disease.

\section{Conclusion}

In conclusion, reported mutations detected within NPM1 of exon 3 showed significant correlation with AML as compared with healthy controls, however such mutations did not affect NES amino acid sequence. No genetic variations were detected in exon 2 of AML patient. In addition, MDR1 gene expression was significantly correlated with chemotherapy induction in AML patients.

\section{Acknowledgement}

The authors are grateful to the College of Biotechnology at Al-Naharin University for offering the space and utilities for accomplishing the project.

\section{Author's Contributions}

Ahmed Abduljabbar Suleiman, Tamadher Abbas Rafaa and Hasan Abdulwahab Jwad: Participated in all experiments designed the research plan and organized the study.

Ali Zaid Al-Saffar: Coordinated the data-analysis and contributed to the writing of the manuscript.

\section{Conflict of Interest}

The contribution of the authors was equal $100 \%$ and the authors declare that there is no competing interest. The project was completely funded by Al-Nahrain University-College of Science.

\section{References}

Almeida, A.M. and F. Ramos, 2016. Acute myeloid leukemia in the older adults. Leukemia Res. Rep., 6: 1-7. DOI: 10.1016/j.lrr.2016.06.001

Baran, Y., B. Gur, P. Kaya, A.U. Ural and F. Avcu et al., 2007. Upregulation of multi drug resistance genes in doxorubicin resistant human acute myelogeneous leukemia cells and reversal of the resistance. Hematology, 12: 511-517. DOI: $10.1080 / 10245330701562535$

ICB, 2014. Iraqi cancer registry. Ministry of Health, Baghdad, Iraq.

Chou, W.C., J.L. Tang, L.I. Lin, M. Yao and W. Tsay et al., 2006. Nucleophosmin mutations in de novo acute myeloid leukemia: The agedependent incidences and the stability during disease evolution. Cancer Res., 66: 3310-3316. DOI: 10.1158/0008-5472.can-05-4316 
Cianfriglia, M., 2013. The biology of MDR1-Pglycoprotein (MDR1-Pgp) in designing functional Antibody Drug Conjugates (ADCs): The experience of gemtuzumab ozogamicin. Ann. Ist. Super Sanita, 49: 150-68. DOI: $10.4415 /$ ann_13_02_07

Ding, L., T.J. Ley, D.E. Larson, $\bar{C}$.A. Miller and D.C. Koboldt et al., 2012. Clonal evolution in relapsed acute myeloid leukaemia revealed by whole-genome sequencing. Nature, 481: 506-510. DOI: $10.1038 /$ nature 10738

Falini, B., N. Bolli, A. Liso, M.P. Martelli and R. Mannucci et al., 2009. Altered nucleophosmin transport in acute myeloid leukaemia with mutated NPM1: Molecular basis and clinical implications. Leukemia, 23: 1731-1743.

Falini, B., I. Nicoletti, M.F. Martelli and C. Mecucci, 2007. Acute myeloid leukemia carrying cytoplasmic/mutated nucleophosmin (NPMc+ AML): Biologic and clinical features. Blood, 109: 874-885. DOI: 10.1182/blood-2006-07-012252

Fletcher, J.I., M. Haber, M.J. Henderson and M.D. Norris, 2010. ABC transporters in cancer: More than just drug efflux pumps. Nat. Rev. Cancer, 10: 147-156. DOI: $10.1038 / \mathrm{nrc} 2789$

Gupta, P.B., C.L. Chaffer and R.A. Weinberg, 2009. Cancer stem cells: Mirage or reality? Nat. Med., 15: 1010-1012. DOI: 10.1038/nm0909-1010

Hamed, N., N. Farahat, M. El Sorady, D. Nafee and S. Barakat, 2011. Clinical significance of sCD86 levels in patients with acute myelogenous leukemia. Alexandria J. Med., 47: 25-30.

DOI: 10.1016/j.ajme.2011.04.002

Herrera, J.E., J.J. Correia, A.E. Jones and M.O. Olson, 1996. Sedimentation analyses of the salt- and divalent metal ion-induced oligomerization of nucleolar protein B23. Biochemistry, 35: 2668-2673.

DOI: $10.1021 /$ bi9523320

Hingorani, K., A. Szebeni and M.O. Olson, 2000. Mapping the functional domains of nucleolar protein B23. J. Biol. Chem., 275: 24451-24457.

DOI: $10.1074 /$ jbc.M003278200

Honoki, K., 2010. Do stem-like cells play a role in drug resistance of sarcomas? Expert Rev. Anticancer Ther., 10: 261-270. DOI: 10.1586/era.09.184

Kazem, A.H., I.L. Mikhael and A.M. Ghanem, 2011. Cytoplasmic Nucleophosmin (cNPM) in acute myeloid leukaemia: Relation to disease characteristics. Alexandria J. Med., 47: 225-235. DOI: 10.1016/j.ajme.2011.07.015

Liu, H., B.C.M. Tan, K.H. Tseng, C.P. Chuang and C.W. Yeh et al., 2007. Nucleophosmin acts as a novel AP2 $\alpha$-binding transcriptional corepressor during cell differentiation. EMBO Rep., 8: 394-400.

DOI: $10.1038 /$ sj.embor.7400909
Longley, D.B. and P.G. Johnston, 2005. Molecular mechanisms of drug resistance. J. Pathol., 205: 275-292. DOI: $10.1002 /$ path. 1706

Raggers, R.J., I. Vogels and G. van Meer, 2001. Multidrug-Resistance P-glycoprotein (MDR1) secretes platelet-activating factor. Biochem. J., 357: 859-865.

Schaich, M., S. Soucek, C. Thiede, G. Ehninger and T. Illmer et al., 2005. MDR1 and MRP1 gene expression are independent predictors for treatment outcome in adult acute myeloid leukaemia. Brit. J. Haematol., 128: 324-332. DOI: $10.1111 /$ j.1365-2141.2004.05319.x

Suzuki, T., H. Kiyoi, K. Ozeki, A. Tomita and S. Yamaji et al., 2005. Clinical characteristics and prognostic implications of NPM1 mutations in acute myeloid leukemia. Blood, 106: 2854-2861. DOI: $10.1182 /$ blood-2005-04-1733

Szankasi, P., M. Jama and D.W. Bahler, 2008. A new DNA-based test for detection of nucleophosmin exon 12 mutations by capillary electrophoresis. J. Molecular Diagnost., 10: 236-241. DOI: $10.2353 /$ jmoldx.2008.070167

Tan, A.Y., D.A. Westerman, D.A. Carney, J.F. Seymour and S. Juneja et al., 2008. Detection of NPM1 exon 12 mutations and FLT3-internal tandem duplications by high resolution melting analysis in normal karyotype acute myeloid leukemia. J. Hematol. Oncol., 1: 10-10. DOI: 10.1186/1756-8722-1-10

Vardiman, J.W., J. Thiele, D.A. Arber, R.D. Brunning and M.J. Borowitz et al., 2009. The 2008 revision of the World Health Organization (WHO) classification of myeloid neoplasms and acute leukemia: Rationale and important changes. Blood, 114: 937-951. DOI: 10.1182/blood-2009-03-209262

Wang, W., A. Budhu, M. Forgues and X.W. Wang, 2005. Temporal and spatial control of nucleophosmin by the Ran-Crm1 complex in centrosome duplication. Nat. Cell Biol., 7: 823-830. DOI: $10.1038 / \mathrm{ncb} 1282$

Webber, B.A., M.M. Cushing and S. Li, 2008. Prognostic significance of flow cytometric immunophenotyping in acute myeloid leukemia. Int. J. Clin. Exp. Pathol., 1: 124-133.

Yu, Y., L.B. Maggi, Jr., S.N. Brady, A.J. Apicelli and M.S. Dai et al., 2006. Nucleophosmin is essential for ribosomal protein L5 nuclear export. Mol. Cell Biol., 26: 3798-3809.

DOI: $10.1128 / \mathrm{mcb} .26 .10 .3798-3809.2006$ 